\title{
On the Generalized Hardy-Rellich Inequalities
}

\author{
T.V. Anoop *, Ujjal Das, Abhishek Sarkar ${ }^{\dagger}$
}

\begin{abstract}
In this article, we look for the weight functions (say $g$ ) that admits the following generalized Hardy-Rellich type inequality:

$$
\int_{\Omega} g(x) u^{2} \mathrm{~d} x \leq C \int_{\Omega}|\Delta u|^{2} \mathrm{~d} x, \forall u \in \mathcal{D}_{0}^{2,2}(\Omega),
$$

for some constant $C>0$, where $\Omega$ is an open set in $\mathbb{R}^{N}$ with $N \geq 1$. We find various classes of such weight functions, depending on the dimension $N$ and the geometry of $\Omega$. Firstly, we use the Muckenhoupt condition for the one dimensional weighted Hardy inequalities and a symmetrization inequality to obtain admissible weights in certain Lorentz-Zygmund spaces. Secondly, using the fundamental theorem of integration we obtain the weight functions in certain weighted Lebesgue spaces. As a consequence of our results, we obtain simple proofs for the embeddings of $\mathcal{D}_{0}^{2,2}(\Omega)$ into certain Lorentz-Zygmund spaces proved by Hansson and later by Brezis and Wainger.
\end{abstract}

Mathematics Subject Classification (2010): 35A23, 46E30, 46E35.

Keywords: Generalized Hardy-Rellich inequality, Muckenhoupt condition, Symmetrization, Lorentz spaces, Lorentz-Zygmund spaces, Exterior domains.

\section{Introduction and Main Results}

In this article, we discuss the generalized Hardy-Rellich inequalities. More precisely, we look for the weight functions $g$ that satisfy the following inequality:

$$
\int_{\Omega} g(x) u^{2} \mathrm{~d} x \leq C \int_{\Omega}|\Delta u|^{2} \mathrm{~d} x, \quad \forall u \in \mathcal{D}_{0}^{2,2}(\Omega),
$$

*corresponding author

${ }^{\dagger}$ The author was supported by the project LO1506 of the Czech Ministry of Education, Youth and Sports. 
where $\Omega$ is an open set in $\mathbb{R}^{N}$ with $N \geq 1, g \in L_{l o c}^{1}(\Omega)$ and $\mathcal{D}_{0}^{2,2}(\Omega)$ is the completion of $\mathcal{C}_{c}^{\infty}(\Omega)$ with respect to $\|\Delta u\|_{L^{2}(\Omega)}$. Depending on the dimension $N$ and on the geometry of $\Omega$, we find various classes of weight function that satisfies (1.1).

The restriction on the dimension is mainly due to the fact that the Beppo-Levi space $\mathcal{D}_{0}^{2,2}(\Omega)$ may not be a function space for a general unbounded open set $\Omega$. For example, when $1 \leq N \leq 4$, Hormander-Lions in [23] showed that $\mathcal{D}_{0}^{2,2}\left(\mathbb{R}^{N}\right)$ contains objects that do not belong to even in the space of distributions. However, when $\Omega$ is an exterior domain we will see that (Remark 3.14) $\mathcal{D}_{0}^{2,2}(\Omega)$ is a well defined function space for any dimension $N$. On the other hand, if $N \geq 5$ or $\Omega$ is bounded, then $\mathcal{D}_{0}^{2,2}(\Omega)$ is always a function space and it is embedded in to certain Lebesgue spaces. Thus depending on $N$, we will be considering various types of $\Omega$ that ensures the Beppo-Levi space $\mathcal{D}_{0}^{2,2}(\Omega)$ is a function space:

(i) for $N \geq 5$ : $\Omega$ is an open set (bounded or unbounded),

(ii) for $2 \leq N \leq 4$ : $\Omega$ is a bounded open set or an exterior domain.

Having made the assumptions on $N$ and $\Omega$, we next look for conditions on $g$ so that (1.1) holds.

First, recall the following classical Hardy-Sobolev inequality:

$$
\int_{\Omega} \frac{|u(x)|^{2}}{|x|^{2}} \mathrm{~d} x \leq\left(\frac{2}{N-2}\right)^{2} \int_{\Omega}|\nabla u|^{2} \mathrm{~d} x, \forall u \in H_{0}^{1}(\Omega),
$$

where $\Omega$ is an open set in $\mathbb{R}^{N}(N \geq 3)$ containing the origin. Many proofs for (1.2) are available in the literature. For an excellent review of this topic we refer to the book [25]. Hardy-Sobolev inequality has been extended and generalized in several directions and for different function spaces. The improved Hardy-Sobolev inequalities are the ones that concerns with replacing the Hardy potential $\frac{1}{|x|^{2}}$ with $\frac{1}{|x|^{2}}+$ lower order radial weights, see $[2,10,18]$ and the references therein. On the other hand, many authors are also interested in generalized Hardy- Sobolev inequalities, i.e., more general weight functions in (1.2) in place of $\frac{1}{|x|^{2}}$. For example, weights in certain Lebesgue spaces [5,27], weak Lebesgue spaces [35] $(N \geq 3)$ and Lorentz-Zygmund spaces [6] ( $N=2$ and $\Omega$ is a bounded). In this article, we study the second order generalization of the Hardy-Sobolev inequality, namely (1.1). For brevity, we make the following definition:

Definition 1.1. A function $g$ that satisfies (1.1) is called an admissible weight.

Notice that the admissibility of $g^{+}$ensures the admissibility of $g$, henceforth in this article, we will be considering nonnegative admissible weight functions. A nonnegative 
admissible function necessarily belongs to $L_{l o c}^{1}(\Omega)$. The following second order generalization (for $N \geq 5$ ) of the classical Hardy-Sobolev inequality is due to Rellich [31]:

$$
\int_{\Omega} \frac{|u(x)|^{2}}{|x|^{4}} \mathrm{~d} x \leq \frac{16}{N^{2}(N-4)^{2}} \int_{\Omega}|\Delta u|^{2} \mathrm{~d} x, \forall u \in W^{2,2}\left(\mathbb{R}^{N}\right) .
$$

Thus $\frac{1}{|x|^{4}}$ is an admissible weight. The authors used the spherical harmonics in [31] to obtain the inequality (1.3), (see Section 7, Chapter 2, page 90-101). Thereafter, many improved Rellich inequalities are proved in the literature, for example see [3,4,19,33,34]. For further readings on the improved Hardy-Sobolev (first order) and Hardy-Rellich inequalities we refer to the monograph [20] and the references therein. The lack of PólyaSzegö type inequality for the second order derivatives is one of the main difficulties in proving the Hardy-Rellich inequality. In general, the Schwarz symmetrization of an $W^{2,2}\left(\mathbb{R}^{N}\right)$ function do not admit the second order weak derivatives, even if they do, the second order derivatives may not satisfy the Pólya-Szegö type inequality, see $[12,28]$ for more discussion on this.

The embeddings of $\mathcal{D}_{0}^{2,2}(\Omega)$ provide admissible weights in the dual of a space associated with the target space in the embedding. Moreover, a finer embedding (a smaller target space) gives a larger class of admissible weights. For example, for $N \geq 5$, the embedding of $\mathcal{D}_{0}^{2,2}\left(\mathbb{R}^{N}\right)$ into the Lebesgue space $L^{2^{* *}}\left(\mathbb{R}^{N}\right)\left(2^{* *}=\frac{2 N}{N-4}\right)$ ensures that $L^{\frac{N}{4}}\left(\mathbb{R}^{N}\right)$ functions are admissible as obtained in [32]. A finer embedding of $\mathcal{D}_{0}^{2,2}(\Omega)$ into the Lorentz space $L^{2^{* *}, 2}(\Omega) \subset L^{2^{* *}}(\Omega)$ is also available, see [28]. The embedding of $\mathcal{D}_{0}^{2,2}(\Omega)$ into a smaller space $L^{2^{* *}, 2}(\Omega)$ provides a bigger class of admissible functions, namely the Lorentz space $L^{\frac{N}{4}, \infty}(\Omega)$. In this article, we present a proof for the admissibility of functions in $L^{\frac{N}{4}, \infty}(\Omega)$ without using the above embedding and then obtain the embedding as a simple consequence of the admissibility. The following theorem is one of our main results:

Theorem 1.2. Let $\Omega$ be an open set in $\mathbb{R}^{N}$ with $N \geq 5$ and $g$ be a nonnegative function.

(i) (A sufficient condition) If $g \in L^{\frac{N}{4}, \infty}(\Omega)$, then $g$ is admissible.

(ii) (A necessary condition) In addition, let $\Omega$ be a ball centered at the origin or entire $\mathbb{R}^{N}$ and $g$ be radial, radially decreasing. Then $g$ is admissible, only if $g$ belongs to $L^{\frac{N}{4}, \infty}(\Omega)$.

Our proofs mainly rely on the Muckenhoupt necessary and sufficient conditions (Theorem 1 and Theorem 2 of [29]) for the one dimensional weighted Hardy inequalities and a pointwise inequality for the symmetrization that obtained in [13] (see (1.14)) using the rearrangement inequality for the convolution due to O'Neil (see [30]). We refer to [16], for similar inequalities for the higher order derivatives. Further, Theorem 1.2 provides a 
simple proof for the embedding of $\mathcal{D}_{0}^{2,2}(\Omega)$ into the Lorentz space $L^{2^{* *}, 2}(\Omega)$ (see Corollary 3.6).

The space $L^{\frac{N}{4}, \infty}(\Omega)$ does not include all the admissible weights. In the next theorem, we exhibit another class of admissible weights. The analogous result for the first order Hardy- Sobolev inequalities is obtained in [14](see Lemma 1.1).

Theorem 1.3. Let $\Omega \subset \mathbb{R}^{N}$ with $N \geq 5$ and let $g$ be a nonnegative function on $\Omega$. If there exists a function $w \in L^{1}\left((0, \infty), r^{3}\right)$ such that $g(x) \leq w(|x|)$ for all $x \in \Omega$, then $g$ is admissible.

The proof of the above theorem is based on the fundamental theorem of integral calculus. Further, we give examples of admissible weights to show that the classes of admissible weights given by Theorem 1.2 and Theorem 1.3 are not contained in one another.

As we mentioned before, when $N=4$, the space $\mathcal{D}_{0}^{2,2}(\Omega)$ may not be a function space for a general unbounded open set $\Omega$. However, for a bounded open set $\Omega$, the Beppo-Levi space $\mathcal{D}_{0}^{2,2}(\Omega)$ coincides with the usual Sobolev space $H_{0}^{2}(\Omega)$. Further,

$$
H_{0}^{2}(\Omega) \hookrightarrow L^{A}(\Omega)
$$

where $L^{A}(\Omega)$ is the Orlicz space generated by the N-function $A(t)=e^{t^{2}}$. Using this embedding, one can show that all the nonnegative functions in the Orlicz space $L \log L(\Omega)$ are admissible. In this case, we use a point wise inequality for the symmetrization and the Muckenhoupt conditions for the one dimensional weighted Hardy inequalities to obtain a bigger class of admissible weights. For a measurable function $g$, we denote its decreasing rearrangement by $g^{*}$ and $g^{* *}(t)=\frac{1}{t} \int_{0}^{t} g^{*}(s) d s$. Now we define

$$
\mathcal{M} \log L(\Omega):=\left\{g \text { measurable }: \sup _{0<t<|\Omega|} t \log \left(\frac{|\Omega|}{t}\right) g^{* *}(t)<\infty\right\} .
$$

$\mathcal{M} \log L(\Omega)$ is a rearrangement invariant Banach function space with the norm

$$
\|g\|_{\mathcal{M} \log L(\Omega)}=\sup _{0<t<|\Omega|} t \log \left(\frac{|\Omega|}{t}\right) g^{* *}(t),
$$

for more on Banach function space see [9]. Now we state our next result.

Theorem 1.4. Let $\Omega$ be a bounded open set in $\mathbb{R}^{4}$ and let $g$ be a nonnegative function.

1. (A sufficient condition) If $g \in \mathcal{M} \log L(\Omega)$, then $g$ is admissible.

2. (A necessary condition) In addition, let $\Omega$ be a ball centered at the origin and let $g$ be radial, radially decreasing. Then $g$ is admissible, only if $g$ belongs to $\mathcal{M} \log L(\Omega)$. 
As a simple consequence of the above theorem we have Corollary 3.12, which gives the embedding of $H_{0}^{2}(\Omega)$ into a Lorentz-Zygmund spaces (finer than the embedding to Orlicz spaces) obtained independently by Brezis and Wainger [11] and Hansson [22].

Next we consider the exterior domains and annular regions in $\mathbb{R}^{N}$ with $2 \leq N \leq 4$. In this case we have the following results:

Theorem 1.5. Let $\Omega=B_{R} \backslash \bar{B}_{1} \subset \mathbb{R}^{N}$ with $1 \leq R \leq \infty$. Let $g$ be a nonnegative function and $w$ be another function such that $g(x) \leq w(|x|)$ for all $x \in \Omega$. If

$$
w \in \begin{cases}L^{1}\left((1, \infty), r^{N+1}\right), & N=3,4 ; R=\infty \\ L^{1}\left((1, \infty), r^{3} \log r\right), & N=2 ; R=\infty \\ L^{1}(1, R), & 2 \leq N \leq 4 ; R<\infty .\end{cases}
$$

then $g$ is admissible.

This article is organized as follows. In Section 2, we briefly discussions the function spaces and other prerequisites which are essential for the development of this article. Section 3 deals with the proof of Theorem 1.2- Theorem 1.5. In appendix, we present some results on Lorentz-Zygmund spaces which we require in this article.

\section{Preliminaries}

In this section, we first describe the symmetrization and some of its properties, then we briefly discuss about the rearrangement invariant function spaces which will appear in this article. In the end, we discuss the Muckenhoupt conditions for the one dimensional weighted Hardy inequalities.

\subsection{Symmetrization}

Let $\Omega \subset \mathbb{R}^{N}$ be a Lebesgue measurable set. Let $\mathcal{M}(\Omega)$ be the set of all extended real valued Lebesgue measurable functions those are finite a.e. in $\Omega$. For $f \in \mathcal{M}(\Omega)$ and for $s>0$, we define $E_{f}(s)=\{x:|f(x)|>s\}$. Then the distribution function $\alpha_{f}$ of $f$ is defined as

$$
\alpha_{f}(s):=\left|E_{f}(s)\right|, \text { for } s>0
$$

where $|A|$ denotes the Lebesgue measure of a set $A \subset \mathbb{R}^{N}$. Now we define the one dimensional decreasing rearrangement $f^{*}$ of $f$ as below:

$$
f^{*}(t):=\left\{\begin{array}{l}
\operatorname{ess} \sup f, \quad t=0 \\
\inf \left\{s>0: \alpha_{f}(s)<t\right\}, t>0 .
\end{array}\right.
$$


The map $f \mapsto f^{*}$ is not sub-additive. However, we obtain a sub-additive function from $f^{*}$, namely the maximal function $f^{* *}$ of $f^{*}$, defined by

$$
f^{* *}(t)=\frac{1}{t} \int_{0}^{t} f^{*}(\tau) d \tau, \quad t>0
$$

The sub-additivity of $f^{* *}$ with respect to $f$ helps us to define norms in certain function spaces.

The Schwarz symmetrization of $f$ is defined by

$$
f^{\star}(x)=f^{*}\left(\omega_{N}|x|^{N}\right), \quad \forall x \in \Omega^{\star},
$$

where $\omega_{N}$ is the measure of the unit ball in $\mathbb{R}^{N}$ and $\Omega^{\star}$ is the open ball centered at the origin with same measure as $\Omega$.

Next we state an important inequality concerning the Schwarz symmetrization, see Theorem 3.2 .10 of [15].

Proposition 2.1 (Hardy-Littlewood inequality). Let $\Omega \subset \mathbb{R}^{N}$ with $N \geq 1$ and $f, g$ be nonnegative measurable functions. Then

$$
\int_{\Omega} f(x) g(x) \mathrm{d} x \leq \int_{\Omega^{\star}} f^{\star}(x) g^{\star}(x) \mathrm{d} x=\int_{0}^{|\Omega|} f^{*}(t) g^{*}(t) \mathrm{d} t .
$$

\subsection{Lorentz spaces}

The Lorentz spaces are refinement of usual Lebesgue spaces introduced by Lorentz himself in [26]. For more details on Lorentz spaces and related results, we refer to the books $[1,15,21]$ and the article [24].

Let $\Omega$ be an open set in $\mathbb{R}^{N}$. Given a function $f \in \mathcal{M}(\Omega)$ and $(p, q) \in[1, \infty) \times[1, \infty]$ we consider the following quantity:

$$
|f|_{(p, q)}:=\left\|t^{\frac{1}{p}-\frac{1}{q}} f^{*}(t)\right\|_{L^{q}((0, \infty))}=\left\{\begin{array}{l}
\left(\int_{0}^{\infty}\left[t^{\frac{1}{p}-\frac{1}{q}} f^{*}(t)\right]^{q} \mathrm{~d} t\right)^{\frac{1}{q}} ; 1 \leq q<\infty, \\
\sup _{t>0} t^{\frac{1}{p}} f^{*}(t) ; q=\infty .
\end{array}\right.
$$

The Lorentz space $L^{p, q}(\Omega)$ is defined as

$$
L^{p, q}(\Omega):=\left\{f \in \mathcal{M}(\Omega):|f|_{(p, q)}<\infty\right\} .
$$

$|f|_{(p, q)}$ is a complete quasi norm on $L^{p, q}(\Omega)$. For $(p, q) \in(1, \infty) \times[1, \infty]$, let

$$
\|f\|_{(p, q)}:=\left\|t^{\frac{1}{p}-\frac{1}{q}} f^{* *}(t)\right\|_{L^{q}((0, \infty))} .
$$

Then $\|f\|_{(p, q)}$ is a norm on $L^{p, q}(\Omega)$ and it is equivalent to the quasinorm $|f|_{(p, q)}$ (see Lemma 3.4.6 of [15]). For the computational simplicity, we use $|f|_{(p, q)}$ instead of $\|f\|_{(p, q)}$. Note that $L^{p, p}(\Omega)=L^{p}(\Omega)$ for $p \in(1, \infty)$ and $L^{p, \infty}(\Omega)$ coincides with the weak- $L^{p}$ space (Marcinkiewicz space) $:=\left\{f \in \mathcal{M}(\Omega): \sup _{s>0} s\left(\alpha_{f}(s)\right)^{\frac{1}{p}}<\infty\right\}$. 


\subsection{Lorentz-Zygmund Space}

Now we briefly sketch an overview of Lorentz-Zygmund spaces. For more details on Lorentz-Zygmund Spaces we refer $[8,9,17]$. For a bounded open set $\Omega \subset \mathbb{R}^{N}, 1 \leq p, q \leq$ $\infty$, and $\alpha \in \mathbb{R}$ we define the following quasinorms:

$$
|f|_{(p, q, \alpha)}=\left\|\left(\log \left(\frac{e|\Omega|}{t}\right)\right)^{\alpha} t^{\frac{1}{p}-\frac{1}{q}} f^{*}(t)\right\|_{L^{q}((0,|\Omega|))} .
$$

Then the Lorentz-Zygmund space $L^{p, q}(\log L)^{\alpha}(\Omega)$ is defined as

$$
L^{p, q}(\log L)^{\alpha}(\Omega):=\left\{f \in \mathcal{M}(\Omega):|f|_{(p, q, \alpha)}<\infty\right\}
$$

For $p, q$ and $\alpha$ as before, let

$$
\|f\|_{(p, q, \alpha)}=\left\|\left(\log \left(\frac{e|\Omega|}{t}\right)\right)^{\alpha} t^{\frac{1}{p}-\frac{1}{q}} f^{* *}(t)\right\|_{L^{q}((0,|\Omega|))} .
$$

For $p>1,\|f\|_{(p, q, \alpha)}$ is a norm on $L^{p, q}(\log L)^{\alpha}(\Omega)$ and it is equivalent to the quasinorm $|f|_{(p, q, \alpha)}$ ( see Corollary 8.2 of [8]). In appendix (Proposition A.3), we provide a proof for the equivalence in the case $p=\infty, q=2$ and $\alpha=-1$.

Note that, $L^{p, q}(\log L)^{0}(\Omega)$ coincides with the Lorentz space $L^{p, q}(\Omega)$. In appendix, we show that as a vector space $L^{1, \infty}(\log L)^{2}(\Omega)$ and $\mathcal{M} \log L(\Omega)$ are same (Proposition A.1). However, the quasinorm $|f|_{(1, \infty, 2)}$ and the norm $\|f\|_{\mathcal{M} \log L}$ are not equivalent.

\subsection{Muckenhoupt Condition}

The following necessary and sufficient conditions (see Theorem 1 and Theorem 2 of [29]) for the one dimensional weighted Hardy inequalities play an important role in our results:

Theorem 2.2 (Muckenhoupt condition). Let $u, v$ be nonnegative measurable functions such that $v>0$. Then for any $a \in(0, \infty]$,

(i) the inequality

$$
\int_{0}^{a}\left|\int_{0}^{s} f(t) \mathrm{d} t\right|^{2} u(s) \mathrm{d} s \leq C \int_{0}^{a}|f(s)|^{2} v(s) \mathrm{d} s,
$$

holds for all measurable function $f$ on $(0, a)$ if and only if

$$
A_{1}:=\sup _{0<t<a}\left(\int_{t}^{a} u(s) \mathrm{d} s\right)\left(\int_{0}^{t} v(s)^{-1} \mathrm{~d} s\right)<\infty .
$$


(ii) the dual inequality

$$
\int_{0}^{a}\left|\int_{s}^{a} f(t) \mathrm{d} t\right|^{2} u(s) \mathrm{d} s \leq C \int_{0}^{a}|f(s)|^{2} v(s) \mathrm{d} s
$$

holds for all measurable function $f$ on $(0, a)$ if and only if

$$
A_{2}:=\sup _{0<t<a}\left(\int_{0}^{t} u(s) \mathrm{d} s\right)\left(\int_{t}^{a} v(s)^{-1} \mathrm{~d} s\right)<\infty .
$$

Remark 2.3. Let $C_{b}^{1}$ and $C_{b}^{2}$ denote the best constants in (2.2) and (2.4) respectively. Then we have the following inequality (see [25])

$$
A_{i} \leq C_{b}^{i} \leq 2 A_{i}, \text { for } i=1,2,
$$

where $A_{i}$ 's are defined in (2.3) and (2.5).

\section{Proof of main theorems}

In this section we prove our main theorems. First, we state an inequality (1.14 of [13]) that plays the role of Pólya-Szegö inequality for the second order derivatives. This inequality is obtained using the rearrangement inequality for the convolution due to O'Neil [30].

Lemma 3.1. For $u \in \mathcal{C}_{c}^{\infty}\left(\mathbb{R}^{N}\right)$ with $N \geq 3$, let $u^{*}$ be the decreasing rearrangement of $u$. Then the following inequality holds:

$$
u^{*}(s) \leq \frac{1}{2(N-2) \omega_{N}^{\frac{2}{N}}}\left(s^{-1+\frac{2}{N}} \int_{0}^{s}|\Delta u|^{*}(t) \mathrm{d} t+\int_{s}^{\infty}|\Delta u|^{*}(t) t^{-1+\frac{2}{N}} \mathrm{~d} t\right), \forall s>0 .
$$

The next lemma is a consequence of the Muckenhoupt condition:

Lemma 3.2. For $N \geq 4$, let $\Omega$ be an open set in $\mathbb{R}^{N}$. In addition, let $\Omega$ be bounded when $N=4$. Then for

$$
g \in X:=\left\{\begin{array}{l}
L^{\frac{N}{4}, \infty}(\Omega), N \geq 5, \\
\mathcal{M} \log L(\Omega), N=4 .
\end{array},\right.
$$

there exists a constant $C=C(N)>0$ such that the following two inequalities hold:

$$
\begin{gathered}
\int_{0}^{|\Omega|} g^{*}(s) s^{-2+\frac{4}{N}}\left(\int_{0}^{s} f(t) \mathrm{d} t\right)^{2} \mathrm{~d} s \leq C\|g\|_{X} \int_{0}^{|\Omega|} f(s)^{2} \mathrm{~d} s \\
\int_{0}^{|\Omega|} g^{*}(s)\left(\int_{s}^{|\Omega|} f(t) t^{-1+\frac{2}{N}} \mathrm{~d} t\right)^{2} \mathrm{~d} s \leq C\|g\|_{X} \int_{0}^{|\Omega|} f(s)^{2} \mathrm{~d} s,
\end{gathered}
$$

for any measurable function $f$ on $(0,|\Omega|)$. 
Proof. For proving (3.2), we set $a=|\Omega|, u(s)=g^{*}(s) s^{-2+\frac{4}{N}}$ and $v(s)=1$ in (2.2). Thus $\int_{0}^{t} v(s)^{-1} \mathrm{~d} s=\int_{0}^{t} \mathrm{~d} s=t$ and

$$
\int_{t}^{a} u(s) \mathrm{d} s=\int_{t}^{|\Omega|} g^{*}(s) s^{-2+\frac{4}{N}} \leq g^{*}(t) \int_{t}^{|\Omega|} s^{-2+\frac{4}{N}} \mathrm{~d} s=\left\{\begin{array}{l}
\frac{N}{N-4} t^{\frac{4}{N}-1} g^{*}(t), N \geq 5 \\
\log \left(\frac{|\Omega|}{t}\right) g^{*}(t), N=4
\end{array}\right.
$$

Therefore,

$$
A_{1}=\sup _{0<t<a}\left(\int_{t}^{a} u(s) \mathrm{d} s\right)\left(\int_{0}^{t} v(s)^{-1} \mathrm{~d} s\right) \leq C\|g\|_{X}<\infty
$$

and hence (3.2) follows from part (i) of Theorem 2.2.

To prove (3.3) we set $a=|\Omega|, u(s)=g^{*}(s)$ and $v(s)=s^{2-\frac{4}{N}}$ in (2.4). Now $\int_{0}^{t} u(s) \mathrm{d} s=$ $\int_{0}^{t} g^{*}(s) \mathrm{d} s=t g^{* *}(t)$ and

$$
\int_{t}^{a} v(s)^{-1} \mathrm{~d} s=\int_{t}^{|\Omega|} s^{-2+\frac{4}{N}} \mathrm{~d} s \leq\left\{\begin{array}{c}
\frac{N}{N-4} t^{\frac{4}{N}-1}, N \geq 5 \\
\log \left(\frac{|\Omega|}{t}\right), N=4
\end{array}\right.
$$

Therefore,

$$
A_{2}=\sup _{0<t<a}\left(\int_{0}^{t} u(s) \mathrm{d} s\right)\left(\int_{t}^{a} v(s)^{-1} \mathrm{~d} s\right) \leq C\|g\|_{X}<\infty .
$$

Hence (3.3) follows from part (ii) of Theorem 2.2.

\subsection{The higher dimension, $N \geq 5$}

In this subsection, we give proofs of Theorem 1.2 and Theorem 1.3.

Proof of Theorem 1.2. (i) A sufficient condition. Let $u \in \mathcal{C}_{c}^{\infty}(\Omega)$. Then by the Hardy-Littlewood inequality (2.1) we have

$$
\int_{\Omega} g(x) u(x)^{2} \mathrm{~d} x \leq \int_{0}^{|\Omega|} g^{*}(s) u^{*}(s)^{2} \mathrm{~d} s .
$$

Further (3.1) gives

$$
\begin{aligned}
\int_{0}^{|\Omega|} g^{*}(s) u^{*}(s)^{2} \mathrm{~d} s \leq & 2 \int_{0}^{|\Omega|} g^{*}(s) s^{-2+\frac{4}{N}}\left(\int_{0}^{s}|\Delta u|^{*}(t) \mathrm{d} t\right)^{2} \mathrm{~d} s \\
& +2 \int_{0}^{|\Omega|} g^{*}(s)\left(\int_{s}^{\infty}|\Delta u|^{*}(t) t^{-1+\frac{2}{N}} \mathrm{~d} t\right)^{2} \mathrm{~d} s .
\end{aligned}
$$

Since $g \in L^{\frac{N}{4}, \infty}(\Omega)$, using Lemma 3.2 we can bound the right hand side of the inequality by $C\|g\|_{\left(\frac{N}{4}, \infty\right)} \int_{0}^{|\Omega|}\left(|\Delta u|^{*}(t)\right)^{2} \mathrm{~d} t$. As $\left\||\Delta u|^{*}\right\|_{L^{2}((0,|\Omega|))}=\|\Delta u\|_{L^{2}(\Omega)}$, (3.4) and (3.5) yields

$$
\int_{\Omega} g(x) u(x)^{2} \mathrm{~d} x \leq C\|g\|_{\left(\frac{N}{4}, \infty\right)} \int_{\Omega}|\Delta u|^{2} \mathrm{~d} x, \forall u \in \mathcal{C}_{c}^{\infty}(\Omega) .
$$


Thus by density of $\mathcal{C}_{c}^{\infty}(\Omega)$ in $\mathcal{D}_{0}^{2,2}(\Omega)$, the above inequality holds for all $u$ in $\mathcal{D}_{0}^{2,2}(\Omega)$ and hence $g$ is admissible.

(ii) A necessary condition. Let $R \in(0, \infty]$ and let $\Omega=B(0 ; R) \subset \mathbb{R}^{N}$ with $N \geq 5$. Let $g: \Omega \rightarrow[0, \infty)$ be a radial and radially decreasing admissible function. We will show that $g \in L^{\frac{N}{4}, \infty}(\Omega)$. For each $r \in(0, R)$, consider the following function:

$$
u_{r}(x)= \begin{cases}(r-|x|)^{2} & ;|x| \leq r \\ 0 & ; \text { otherwise. }\end{cases}
$$

By differentiating twice, we get

$$
\Delta u_{r}(x)= \begin{cases}2 N-(2 N-2) \frac{r}{|x|} & ;|x|<r \\ 0 & ; \text { otherwise. }\end{cases}
$$

Now

$$
\begin{aligned}
\int_{\Omega}\left|\Delta u_{r}\right|^{2} \mathrm{~d} x & =\int_{B_{r}}\left|\Delta u_{r}\right|^{2} \mathrm{~d} x=\int_{B_{r}}\left[2 N-(2 N-2) \frac{r}{|x|}\right]^{2} \mathrm{~d} x \\
& \leq 2\left[4 N^{2} \omega_{N} r^{N}+(2 N-2)^{2} r^{2} \int_{B_{r}} \frac{1}{|x|^{2}} \mathrm{~d} x\right] \\
& \leq C_{1}\left[r^{N}+r^{2} \int_{0}^{r} s^{N-3} \mathrm{~d} s\right] \leq C_{2} r^{N},
\end{aligned}
$$

where $C_{1}, C_{2}$ are constants that depends only on $N$. Thus for each $r \in(0, R), u_{r} \in$ $\mathcal{D}_{0}^{2,2}(\Omega)$. Furthermore, by the admissibility of $g$, we have

$$
\int_{\Omega} g(x) u_{r}^{2} \mathrm{~d} x \leq C \int_{\Omega}\left|\Delta u_{r}\right|^{2} \mathrm{~d} x, \forall r \in(0, R) .
$$

Since $g$ is radial and radially decreasing, the left hand side of the above inequality can be estimated as below:

$$
\begin{aligned}
\int_{\Omega} g(x) u_{r}^{2} \mathrm{~d} x & \geq \int_{B_{\frac{r}{2}}} g(|x|) u_{r}^{2} \mathrm{~d} x \geq\left(r-\frac{r}{2}\right)^{4} \int_{B_{\frac{r}{2}}} g(|x|) \mathrm{d} x \\
& =\left(\frac{r}{2}\right)^{4} \int_{B_{\frac{r}{2}}} g^{\star}(x) \mathrm{d} x=\left(\frac{r}{2}\right)^{4} \int_{0}^{\omega_{N}\left(\frac{r}{2}\right)^{N}} g^{*}(s) \mathrm{d} s .
\end{aligned}
$$

From (3.7), (3.8) and (3.9), we obtain

$$
\left(\frac{r}{2}\right)^{4} \int_{0}^{\omega_{N}\left(\frac{r}{2}\right)^{N}} g^{*}(s) \mathrm{d} s \leq C C_{2} r^{N} .
$$

Now by setting $\omega_{N}\left(\frac{r}{2}\right)^{N}=t$ and since $0<r<R$ is arbitrary, we conclude that

$$
\sup _{t \in\left(0, \frac{|\Omega|}{2^{N}}\right)} t^{\frac{4}{N}} g^{* *}(t) \leq C_{3}
$$

As $t^{\frac{4}{N}} g^{* *}(t)$ is bounded on $\left(\frac{|\Omega|}{2^{N}},|\Omega|\right), g$ must belong to $L^{\frac{N}{4}, \infty}(\Omega)$. 
Remark 3.3. Let $C_{R}$ be the best constant in (1.1). Then from (3.1), (2.6) and Lemma 3.2 one can deduce that

$$
C_{R} \leq \frac{N}{(N-4)(N-2)^{2} \omega_{N}^{\frac{4}{N}}}\|g\|_{\left(\frac{N}{4}, \infty\right)}
$$

Example 3.4. For $\alpha \in(0, N)$ and $R \in(0, \infty]$ let $g(x)=\frac{1}{|x|^{\alpha}}, x \in B(0 ; R)$. It is easy to calculate

$$
g^{*}(t)=\left\{\begin{array}{ll}
\left(\frac{\omega_{N}}{t}\right)^{\frac{\alpha}{N}} & 0<t<\omega_{N} R^{N}, \\
0 & t \geq \omega_{N} R^{N} .
\end{array} \quad g^{* *}(t)= \begin{cases}\frac{N}{N-\alpha}\left(\frac{\omega_{N}}{t}\right)^{\frac{\alpha}{N}} & 0<t<\omega_{N} R^{N}, \\
0 & t \geq \omega_{N} R^{N}\end{cases}\right.
$$

Therefore,

$$
g \in L^{\frac{N}{4}, \infty}(B(0 ; R)) \text { with }\left\{\begin{array}{l}
R<\infty \text { if and only if } \quad \alpha \leq 4 \\
R=\infty \text { if and only if } \quad \alpha=4
\end{array}\right.
$$

Remark 3.5. From the above example, it is clear that $g(x)=\frac{1}{|x|^{4}}$ belongs to $L^{\frac{N}{4}, \infty}\left(\mathbb{R}^{N}\right)$ and $\|g\|_{\left(\frac{N}{4}, \infty\right)}=\frac{N \omega_{N}^{\frac{4}{N}}}{N-4}$. Thus the Hardy-Rellich inequality (1.3) follows easily from part (i) of Theorem 1.2. Further, the best constant in (1.3) equals to $\frac{16}{(N-4)^{2} N^{2}}$ which is bounded by the constant $\frac{N^{2}}{(N-4)^{2}(N-2)^{2}}$ given by Remark 3.3.

As a consequence of the sufficiency part of Theorem 1.2, we have a simple proof for the following Lorentz-Sobolev embedding:

Corollary 3.6. Let $\Omega \subset \mathbb{R}^{N}$ is an open set and $N \geq 5$. Then we have the following embedding:

$$
D_{0}^{2,2}(\Omega) \hookrightarrow L^{2^{* *}, 2}(\Omega), \text { where } 2^{* *}=\frac{2 N}{N-4} .
$$

Proof. Without loss of generality we may assume $\Omega=\mathbb{R}^{N}$ (for a general domain $\Omega$, the result will follow by considering the zero extension to $\mathbb{R}^{N}$ ). By (3.6), for each $g \in$ $L^{\frac{N}{4}, \infty}\left(\mathbb{R}^{N}\right)$ we have

$$
\int_{0}^{\infty} g^{*}(t)\left(u^{*}(t)\right)^{2} \mathrm{~d} t \leq C\|g\|_{\left(\frac{N}{4}, \infty\right)} \int_{\mathbb{R}^{N}}|\Delta u|^{2} \mathrm{~d} x, \forall u \in \mathcal{D}_{0}^{2,2}\left(\mathbb{R}^{N}\right) .
$$

In particular, if we choose $g(x)=\frac{1}{|x|^{4}}$, then $g^{*}(t)=\left(\frac{\omega_{N}}{t}\right)^{\frac{4}{N}}$ and $\|g\|_{\left(\frac{N}{4}, \infty\right)}=\frac{N \omega_{N}^{\frac{4}{N}}}{N-4}$. Now by substituting in the above inequality, we get

$$
\int_{0}^{\infty} t^{-\frac{4}{N}}\left(u^{*}(t)\right)^{2} \mathrm{~d} t \leq C_{1} \int_{\mathbb{R}^{N}}|\Delta u|^{2} \mathrm{~d} x, \forall u \in \mathcal{D}_{0}^{2,2}\left(\mathbb{R}^{N}\right),
$$


where $C_{1}$ is a constant that depends only on $N$. Since $\int_{0}^{\infty} t^{-\frac{4}{N}}\left(u^{*}(t)\right)^{2} \mathrm{~d} t=|u|_{\left(2^{* *}, 2\right)}^{2}$ is equivalent to $\|u\|_{\left(2^{* *}, 2\right)}^{2}$, we obtain the required embedding

$$
\|u\|_{\left(2^{* *}, 2\right)}^{2} \leq C_{2} \int_{\mathbb{R}^{N}}|\Delta u|^{2} \mathrm{~d} x, \forall u \in \mathcal{D}_{0}^{2,2}\left(\mathbb{R}^{N}\right) .
$$

The following lemma is needed for the proofs of Theorems 1.3 and 1.5.

Lemma 3.7. For $u \in C_{c}^{\infty}\left(\mathbb{R}^{N}\right)$, the following inequality holds:

$$
\int_{0}^{\infty} \int_{\mathbb{S}^{N-1}} r^{N-1}\left|\frac{\partial^{2} u}{\partial t^{2}}(r, w)\right|^{2} \mathrm{~d} S_{w} \mathrm{~d} r \leq \int_{\mathbb{R}^{N}}|\Delta u|^{2} \mathrm{~d} x
$$

Proof. Observe that

$$
\frac{\partial u}{\partial \eta}=\nabla u \cdot \eta \text { and } \frac{\partial^{2} u}{\partial \eta^{2}}=\nabla(\nabla u \cdot \eta) \cdot \eta=\sum_{i=1}^{N} \sum_{j=1}^{N} \frac{\partial^{2} u}{\partial x_{i} \partial x_{j}} \eta_{i} \eta_{j} .
$$

Further, have the following inequality for an $N \times N$ real matrix $A=\left(a_{i j}\right)$ and $x \in \mathbb{R}^{N}$ :

$$
|\langle A x, x\rangle|^{2} \leq\left|\sum_{i=1}^{N} \sum_{j=1}^{N} a_{i j} x_{i} x_{j}\right|^{2} \leq\left(\sum_{i=1}^{N} \sum_{j=1}^{N} a_{i j}^{2}\right)\left(\sum_{i=1}^{N} x_{i}^{2}\right)\left(\sum_{j=1}^{N} x_{j}^{2}\right) .
$$

Now by writing $x=(r, \omega) \in(0, \infty) \times \mathbb{S}^{N-1}$ for $x \in \mathbb{R}^{N} \backslash\{0\}$, and using (3.10), we obtain

$$
\begin{aligned}
\int_{0}^{\infty} \int_{\mathbb{S}^{N-1}}\left|\frac{\partial^{2} u}{\partial r^{2}}(r, \omega)\right|^{2} r^{N-1} \mathrm{~d} S_{\omega} \mathrm{d} r & =\int_{\mathbb{R}^{N}}\left|\frac{\partial^{2} u}{\partial r^{2}}\right|^{2} \mathrm{~d} x=\int_{\mathbb{R}^{N}}\left(\sum_{i=1}^{N} \sum_{j=1}^{N} \frac{\partial^{2} u}{\partial x_{i} \partial x_{j}} \frac{x_{i}}{|x|} \frac{x_{j}}{|x|}\right)^{2} \mathrm{~d} x \\
& \leq \int_{\mathbb{R}^{N}} \sum_{i=1}^{N} \sum_{j=1}^{N}\left(\frac{\partial^{2} u}{\partial x_{i} \partial x_{j}}\right)^{2} \mathrm{~d} x=\int_{\mathbb{R}^{N}}|\Delta u|^{2} \mathrm{~d} x
\end{aligned}
$$

and this concludes the proof.

Next we prove Theorem 1.3.

Proof of Theorem 1.3. For $x \in \mathbb{R}^{N} \backslash\{0\}$, using the polar coordinates, we write $x=$ $(r, \omega) \in(0, \infty) \times \mathbb{S}^{N-1}$. Thus for $u \in \mathcal{C}_{c}^{\infty}\left(\mathbb{R}^{N}\right)$,

$$
\begin{aligned}
u(r, \omega) & =-\int_{r}^{\infty} \frac{\partial u}{\partial t}(t, \omega) \mathrm{d} t=-r \frac{\partial u}{\partial t}(r, \omega)+\int_{r}^{\infty} t \frac{\partial^{2} u}{\partial t^{2}}(t, \omega) \mathrm{d} t \\
& =\int_{r}^{\infty}(t-r) \frac{\partial^{2} u}{\partial t^{2}}(t, \omega) \mathrm{d} t .
\end{aligned}
$$


Hence

$$
|u(r, \omega)| \leq \int_{r}^{\infty} t\left|\frac{\partial^{2} u}{\partial t^{2}}(t, \omega)\right| \mathrm{d} t=\int_{r}^{\infty} t t^{\frac{1-N}{2}} t^{\frac{N-1}{2}}\left|\frac{\partial^{2} u}{\partial t^{2}}(t, \omega)\right| \mathrm{d} t .
$$

Now by Hölder inequality, we get

$$
\begin{aligned}
|u(r, \omega)|^{2} & \leq\left(\int_{r}^{\infty} t^{2} t^{1-N} \mathrm{~d} t\right)\left(\int_{r}^{\infty} t^{N-1}\left|\frac{\partial^{2} u}{\partial t^{2}}(t, \omega)\right|^{2} \mathrm{~d} t\right) \\
& =\frac{1}{N-4} r^{4-N} \int_{r}^{\infty} t^{N-1}\left|\frac{\partial^{2} u}{\partial t^{2}}(t, \omega)\right|^{2} \mathrm{~d} t .
\end{aligned}
$$

Multiply both sides of $(3.12)$ by $w(r)$ and integrate over $\mathbb{S}^{N-1}$ to obtain

$$
\begin{aligned}
\int_{\mathbb{S}^{N-1}}|u(r, \omega)|^{2} w(r) \mathrm{d} S_{\omega} & \leq \frac{1}{N-4} r^{4-N} w(r) \int_{0}^{\infty} \int_{\mathbb{S}^{N-1}} t^{N-1}\left|\frac{\partial^{2} u}{\partial t^{2}}(t, \omega)\right|^{2} \mathrm{~d} S_{\omega} \mathrm{d} t \\
& \leq \frac{1}{N-4} r^{4-N} w(r)\left(\int_{\mathbb{R}^{N}}|\Delta u|^{2} \mathrm{~d} x\right),
\end{aligned}
$$

where the last inequality follows from Lemma 3.7. Finally, multiplying both the sides of (3.13) by $r^{N-1}$ and integrating over $(0, \infty)$ with respect to $r$ yields:

$$
\int_{\mathbb{R}^{N}} w(|x|) u^{2} \mathrm{~d} x \leq \frac{N \omega_{N}}{N-4}\left(\int_{0}^{\infty} w(r) r^{3} \mathrm{~d} r\right) \int_{\mathbb{R}^{N}}|\Delta u|^{2} \mathrm{~d} x .
$$

In particular, as $g(x) \leq w(|x|)$ we have

$$
\int_{\Omega} g(x) u^{2} \mathrm{~d} x \leq \frac{N \omega_{N}}{N-4}\left(\int_{0}^{\infty} w(r) r^{3} \mathrm{~d} r\right) \int_{\Omega}|\Delta u|^{2} \mathrm{~d} x, \forall u \in \mathcal{C}_{c}^{\infty}(\Omega) .
$$

Now by density of $\mathcal{C}_{c}^{\infty}(\Omega)$, the above inequality holds for all $u \in \mathcal{D}_{0}^{2,2}(\Omega)$ and hence $g$ is admissible.

Observe that, we have two different set of conditions for the admissibility from Theorem 1.2 and Theorem 1.3. Next examples show that these two conditions are independent, i.e., one does not imply the other.

Example 3.8. Let $\Omega=\mathbb{R}^{N}$ with $N \geq 5$ and let $\beta \in\left(\frac{4}{N}, 1\right)$. Consider

$$
g_{1}(x)= \begin{cases}(|x|-1)^{-\beta}, & 1<|x| \leq 2 \\ 0, & \text { otherwise }\end{cases}
$$

We can compute the distribution function $\alpha_{g_{1}}$ and the one dimensional decreasing rearrangement $g_{1}^{*}$ as below:

$$
\alpha_{g_{1}}(s)= \begin{cases}\omega_{N} 2^{N}-\omega_{N} & , 0 \leq s<1, \\ \omega_{N}\left(s^{-\frac{1}{\beta}}+1\right)^{N}-\omega_{N} & , s \geq 1,\end{cases}
$$




$$
g_{1}^{*}(t)= \begin{cases}0 & , t>\omega_{N}\left(2^{N}-1\right) \\ \left(\left(\frac{t}{\omega_{N}}+1\right)^{\frac{1}{N}}-1\right)^{-\beta}, & t \leq \omega_{N}\left(2^{N}-1\right) .\end{cases}
$$

Hence, for $t \leq \omega_{N}\left(2^{N}-1\right)$,

$$
t^{\frac{4}{N}} g_{1}^{*}(t)=t^{\frac{4}{N}}\left(\left(\frac{t}{\omega_{N}}+1\right)^{\frac{1}{N}}-1\right)^{-\beta} \geq t^{\frac{4}{N}}\left(\left(\frac{t}{\omega_{N}}+1\right)-1\right)^{-\beta}=t^{\frac{4}{N}}\left(\frac{t}{\omega_{N}}\right)^{-\beta}
$$

Since $\beta>\frac{4}{N}, \sup _{t \in(0, \infty)} t^{\frac{4}{N}} g_{1}^{*}(t)=\infty$ and hence $g \notin L^{\frac{N}{4}, \infty}\left(\mathbb{R}^{N}\right)$.

Let $w(r)=(r-1)^{-\beta} \chi_{(1,2)}(r)$. Clearly $g_{1}(x) \leq w(|x|), \forall x \in \mathbb{R}^{N}$ and since $\beta<1$,

$$
\int_{0}^{\infty} w(r) r^{3} \mathrm{~d} r=\int_{1}^{2}(r-1)^{-\beta} r^{3} \mathrm{~d} r \leq 8 \int_{0}^{1} s^{-\beta} \mathrm{d} s<\infty .
$$

Thus $g_{1}$ is admissible by Theorem 1.3 .

Example 3.9. Let $g_{2}(x)=\frac{1}{|x|^{4}}, x \in \mathbb{R}^{N}$ with $N \geq 5$. By Example $3.4, g_{2} \in L^{\frac{N}{4}, \infty}\left(\mathbb{R}^{N}\right)$ and hence admissible by Theorem 1.2. Let $w$ be a function on $(0, \infty)$ such that $g(x) \leq$ $w(|x|)$. Then

$$
\int_{0}^{\infty} w(r) r^{3} \geq \int_{0}^{\infty} r^{-4} \times r^{3} \mathrm{~d} r=\infty
$$

Thus $g_{2}$ does not satisfy the assumptions of Theorem 1.3.

Remark 3.10. The above examples shows that the the sufficient conditions given by Theorem 1.2 and Theorem 1.3 are independent. The question whether these conditions exhaust all the admissible weights or not is open.

Remark 3.11. There are admissible weights whose Schwarz symmetrization are not admissible. For example, the Schwarz symmetrization $g_{1}^{\star}$ of $g_{1}$ does not belong to $L^{\frac{N}{4}, \infty}\left(\Omega^{\star}\right)$ and hence by part of (ii) of Theorem $1.2, g_{1}^{\star}$ can not be an admissible weight.

\subsection{The critical dimension $(N=4)$ and the lower dimensions $(N=1,2$ and 3$)$}

Now we consider the cases $\Omega$ is a bounded open set or an exterior domain. In either cases, the space $\mathcal{D}_{0}^{2,2}(\Omega)$ is a well defined function space. First we give a proof of Theorem 1.4.

Proof of Theorem 1.4. (i) A sufficient condition. The proof follows in the same line as in the proof of Theorem 1.2. Let $u \in \mathcal{C}_{c}^{\infty}(\Omega)$. Then by the Hardy-Littlewood inequality (2.1) we have

$$
\int_{\Omega} g(x) u(x)^{2} \mathrm{~d} x \leq \int_{0}^{|\Omega|} g^{*}(s) u^{*}(s)^{2} \mathrm{~d} s .
$$


Further, using (3.1) we have

$$
\begin{aligned}
\int_{0}^{|\Omega|} g^{*}(s) u^{*}(s)^{2} \mathrm{~d} s & \leq 2 \int_{0}^{|\Omega|} g^{*}(s) s^{-1}\left(\int_{0}^{s}|\Delta u|^{*}(t) \mathrm{d} t\right)^{2} \mathrm{~d} s \\
& +2 \int_{0}^{|\Omega|} g^{*}(s)\left(\int_{s}^{\infty}|\Delta u|^{*}(t) t^{\frac{1}{2}} \mathrm{~d} t\right)^{2} \mathrm{~d} s \\
& \leq C\|g\|_{\mathcal{M} \log L(\Omega)} \int_{0}^{|\Omega|}\left(|\Delta u|^{*}(t)\right)^{2} \mathrm{~d} t
\end{aligned}
$$

where the last inequality follows from Lemma 3.2, as $g \in \mathcal{M} \log L(\Omega)$. From (3.14) and (3.15), we get

$$
\int_{\Omega} g(x) u(x)^{2} \mathrm{~d} x \leq \int_{0}^{|\Omega|} g^{*}(s) u^{*}(s)^{2} \mathrm{~d} s \leq C\|g\|_{\mathcal{M} \log L(\Omega)} \int_{\Omega}|\Delta u|^{2} \mathrm{~d} x,
$$

Thus by density, the above inequality holds for all $u$ in $\mathcal{D}_{0}^{2,2}(\Omega)$ and hence $g$ is admissible. (ii) A necessary condition. Let $R \in(0, \infty)$ and let $\Omega=B(0 ; R) \subset \mathbb{R}^{4}$. Let $g$ be a nonnegative, radial and radially decreasing admissible function on $\Omega$. To show $g \in \mathcal{M} \log L$, for each $r \in(0, R)$, we consider the following test function:

$$
u_{r}(x)= \begin{cases}\frac{1}{e^{2}}\left(\log \left(\frac{R}{r}\right)\right)^{2}, & |x| \leq r \\ \left(\log \left(\frac{R}{|x|}\right)\right)^{2} \Phi_{r}(x), & r<|x|<R\end{cases}
$$

where $\Phi_{r}(x)=\exp \left(-\frac{2 \log \left(\frac{R}{\mid x}\right)}{\log \left(\frac{R}{r}\right)}\right)$. In our computations we use the notation $D_{i} \equiv \frac{\partial}{\partial x_{i}}$ and $D_{i i} \equiv \frac{\partial^{2}}{\partial x_{i}^{2}}$. For $r \leq|x| \leq R$, noting that $D_{i} \Phi_{r}(x)=\frac{2 x_{i}}{|x|^{2} \log \left(\frac{R}{r}\right)} \Phi_{r}(x)$ and $D_{i} \log \left(\frac{R}{|x|}\right)=-\frac{x_{i}}{|x|^{2}}$, we compute the derivatives of $u_{r}$ as below:

$$
D_{i} u_{r}(x)=2 \Phi_{r}(x) \frac{x_{i}}{|x|^{2}} \log \left(\frac{R}{|x|}\right)\left[\frac{\log \left(\frac{R}{|x|}\right)}{\log \left(\frac{R}{r}\right)}-1\right] .
$$

Furthermore,

$$
\begin{aligned}
D_{i i}^{2} u_{r}(x)= & \Phi_{r}(x)\left\{\frac{4 x_{i}^{2}}{|x|^{4}} \frac{\log \left(\frac{R}{|x|}\right)}{\log \left(\frac{R}{r}\right)}+2\left(-\frac{2 x_{i}^{2}}{|x|^{4}}+\frac{1}{|x|^{2}}\right) \log \left(\frac{R}{|x|}\right)-\frac{2 x_{i}^{2}}{|x|^{4}}\right\}\left[\frac{\log \left(\frac{R}{|x|}\right)}{\log \left(\frac{R}{r}\right)}-1\right] \\
& -2 \Phi_{r}(x) \frac{x_{i}^{2}}{|x|^{4}} \frac{\log \left(\frac{R}{|x|}\right)}{\log \left(\frac{R}{r}\right)} .
\end{aligned}
$$

Thus for $r \leq|x| \leq R$,

$$
\Delta u_{r}=\Phi_{r}(x)\left\{\frac{4 \log \left(\frac{R}{|x|}\right)}{|x|^{2} \log \left(\frac{R}{r}\right)}+\frac{4}{|x|^{2}} \log \left(\frac{R}{|x|}\right)-\frac{2}{|x|^{2}}\right\}\left[\frac{\log \left(\frac{R}{|x|}\right)}{\log \left(\frac{R}{r}\right)}-1\right]-2 \Phi_{r}(x) \frac{1}{|x|^{2}} \frac{\log \left(\frac{R}{|x|}\right)}{\log \left(\frac{R}{r}\right)} .
$$


Observe that $\Phi_{r}(x) \leq 1, \log \left(\frac{R}{|x|}\right) \leq \log \left(\frac{R}{r}\right)$ and $1 \leq \log \left(\frac{R}{r}\right)$ for $r \leq \frac{R}{e}$. Hence

$$
\left|\Delta u_{r}(x)\right| \leq \frac{16}{|x|^{2}} \log \left(\frac{R}{|x|}\right)+\frac{4}{|x|^{2}}+\frac{2}{|x|^{2}} \log \left(\frac{R}{|x|}\right) \leq \frac{18}{|x|^{2}} \log \left(\frac{R}{|x|}\right)+\frac{4}{|x|^{2}}
$$

Thus for $r<\frac{R}{e}$, we have

$$
\begin{aligned}
\int_{\Omega}\left|\Delta u_{r}(x)\right|^{2} & \leq C_{1} \int_{\Omega \backslash B(0, r)}\left[\frac{1}{|x|^{4}} \log \left(\frac{R}{|x|}\right)^{2}+\frac{1}{|x|^{4}}\right] \mathrm{d} x \\
& \leq C_{1}\left\{\left[\log \left(\frac{R}{r}\right)\right]^{3}+\log \left(\frac{R}{r}\right)\right\} \leq C_{1}\left[\log \left(\frac{R}{r}\right)\right]^{3},
\end{aligned}
$$

where $C_{1}$ is a positive constant independent of $r$. Notice that $u_{r}$ is a $C^{1}$ function such that $u_{r}$ and $\nabla u_{r}$ vanish when $|x|=R$, hence $u_{r} \in H_{0}^{2}(\Omega)$. Further, as $g$ is radial, radially decreasing, we easily obtain the following estimate:

$$
\int_{\Omega} g(x) u_{r}^{2}(x) \mathrm{d} x \geq \int_{B(0, r)} g(x) u_{r}(x)^{2} \mathrm{~d} x=\left[\frac{1}{e^{2}} \log \left(\frac{R}{r}\right)\right]^{4} \int_{0}^{\omega_{4} r^{4}} g^{*}(s) d s
$$

Now the admissibility of $g$ together with (3.16) and (3.17) yields

$$
\log \left(\frac{R}{r}\right) \int_{0}^{\omega_{4} r^{4}} g^{*}(s) d s \leq C, \quad \forall r \in\left(0, \frac{R}{e}\right) .
$$

By taking $t=\omega_{4} r^{4}$, we get

$$
\frac{1}{4} \log \left(\frac{|\Omega|}{t}\right) \int_{0}^{t} g^{*}(s) d s \leq C, \quad \forall t \in\left(0, \frac{|\Omega|}{e^{4}}\right) .
$$

Since $\operatorname{tg}^{* *}(t) \log \left(\frac{|\Omega|}{t}\right)$ is bounded on $\frac{|\Omega|}{e^{4}} \leq t \leq|\Omega|$, from the above inequality we conclude that

$$
\sup _{t \in(0,|\Omega|)} t g^{* *}(t) \log \left(\frac{|\Omega|}{t}\right)<\infty
$$

Hence $g \in \mathcal{M} \log L(\Omega)$.

As a corollary of the sufficiency part of our previous theorem, we give a simple alternate proof for the embedding of $H_{0}^{2}(\Omega)$ into the Lorentz-Zygmund space $L^{\infty, 2}(\log L)^{-1}(\Omega)$ obtained independently by Brezis and Wainger [11] and Hansson [22].

Corollary 3.12. Let $\Omega \subset \mathbb{R}^{4}$ is an open bounded set. Then we have the following embedding:

$$
H_{0}^{2}(\Omega) \hookrightarrow L^{\infty, 2}(\log L)^{-1}(\Omega)
$$


Proof. First, assume that $\Omega$ is a ball of radious $R$ with center at the origin. Let $X=$ $\mathcal{M} \log L(\Omega)$. For each $g \in X,(3.15)$ gives,

$$
\int_{0}^{|\Omega|} g^{*}(t)\left(u^{*}(t)\right)^{2} \mathrm{~d} t \leq C\|g\|_{X} \int_{\Omega}|\Delta u|^{2} \mathrm{~d} x, \forall u \in H_{0}^{2}(\Omega) .
$$

Let $g_{1}(x)=\left[|x|^{2} \log \left(\left(\frac{R}{|x|}\right)^{4} e\right)\right]^{-2}, x \in \Omega$. We calculate, $g_{1}^{*} g_{1}^{* *}(t)=\frac{\omega_{4}}{t\left(\log \frac{e|\Omega|}{t}\right)}, t \in(0,|\Omega|)$. Therefore, $g_{1} \in X$ and $\left\|g_{1}\right\|_{X}=\omega_{4}$. Thus by the above inequality we have

$$
\int_{0}^{|\Omega|} \frac{\left(u^{*}(t)\right)^{2}}{t\left[\log \left(\frac{e|\Omega|}{t}\right)\right]^{2}} \mathrm{~d} t \leq C_{1} \int_{\Omega}|\Delta u|^{2} \mathrm{~d} x, \forall u \in H_{0}^{2}(\Omega) .
$$

The left hand side of the above inequality is equivalent to $\|u\|_{L^{\infty, 2}(\log L)^{-1}(\Omega)}^{2}$ (Proposition A.3). Therefore,

$$
\|u\|_{L^{\infty, 2}(\log L)^{-1}(\Omega)}^{2} \leq C_{2} \int_{\Omega}|\Delta u|^{2} \mathrm{~d} x, \forall u \in H_{0}^{2}(\Omega) .
$$

Now for a general bounded set $\Omega$, there exists $R>0$ such that $\Omega \subset B(0, R)$. In this case, we obtain the required embedding by considering the above inequality for the zero extension to $B(0, R)$.

Remark 3.13. For a bounded open set, we have the following continuous inclusions:

$$
L^{\infty, 2}(\log L)^{-1}(\Omega) \hookrightarrow L_{e^{t^{2}-1}}(\Omega) \hookrightarrow L^{p}(\Omega), 1 \leq p<\infty
$$

Thus the above embedding gives the classical Sobolev embedding and Adams' embedding:

$$
H_{0}^{2}(\Omega) \hookrightarrow L^{p}(\Omega), 1 \leq p<\infty ; \quad H_{0}^{2}(\Omega) \hookrightarrow L_{e^{t^{2}-1}}(\Omega)
$$

Next we give a proof of Theorem 1.5 for the cases $N=2,3,4$.

Proof of Theorem 1.5 for the cases $N=2,3,4$. As before, for $x \in B_{R} \backslash B_{1}$, we write $x=(r, \omega) \in(1, R) \times \mathbb{S}^{N-1}$. For $u \in \mathcal{C}_{c}^{\infty}(\Omega)$, we use the fundamental theorem of calculus to get

$$
u(r, \omega)=\int_{1}^{r} \frac{\partial u}{\partial t}(t, \omega) \mathrm{d} t .
$$

As in the proof of Theorem 1.3, we deduce

$$
u(r, \omega)=\int_{1}^{r}(r-t) \frac{\partial^{2} u}{\partial t^{2}}(t, \omega) \mathrm{d} t=\int_{1}^{r}(r-t) t^{-\frac{N-1}{2}} t^{\frac{N-1}{2}} \frac{\partial^{2} u}{\partial t^{2}}(t, \omega) \mathrm{d} t
$$


Now Hölder inequality yields

$$
|u(r, \omega)|^{2} \leq r^{2}\left(\int_{1}^{r} t^{-(N-1)} \mathrm{d} t\right)\left(\int_{1}^{r} t^{N-1}\left|\frac{\partial^{2} u}{\partial t^{2}}(t, \omega)\right|^{2} d t\right) .
$$

Multiply the above inequality by $r^{N-1} w(r)$ and integrate over $\mathbb{S}^{N-1} \times(1, R)$ and use Lemma 3.7 to obtain

$$
\begin{aligned}
\int_{B_{R} \backslash B_{1}} w(|x|) u^{2} \mathrm{~d} x & \leq\left(\int_{1}^{R}\left[\int_{1}^{r} t^{1-N} \mathrm{~d} t\right] r^{N+1} w(r) \mathrm{d} r\right) \int_{\mathbb{S}^{N-1}} \int_{1}^{R} t^{N-1}\left|\frac{\partial^{2} u}{\partial t^{2}}(t, \omega)\right|^{2} d t \mathrm{~d} S_{\omega} \\
& \leq I \times\left(\int_{B_{R} \backslash B_{1}}|\Delta u|^{2} \mathrm{~d} x\right),
\end{aligned}
$$

where $I=\left(\int_{1}^{R} r^{N+1}\left[\int_{1}^{r} t^{1-N} \mathrm{~d} t\right] w(r) \mathrm{d} r\right)$. Notice that

$$
I \leq\left\{\begin{array}{l}
\int_{1}^{\infty} r^{N+1} w(r) \mathrm{d} r, \quad N=3,4 ; R=\infty \\
\int_{1}^{\infty} r^{3}(\log r) w(r) \mathrm{d} r, \quad N=2 ; R=\infty \\
\int_{1}^{R} w(r) \mathrm{d} r, \quad 2 \leq N \leq 4 ; R<\infty .
\end{array}\right.
$$

Therefore, the assumptions on $g$ together with (3.18) and (3.19) gives the admissibility of $g$.

Remark 3.14. Let $f \in L^{1}(1, \infty)$ and $f \geq 0$. Then by Theorem 1.5, we have the following embeddings:

$$
\mathcal{D}_{0}^{2,2}\left(R^{N} \backslash \overline{B_{1}}\right) \hookrightarrow\left\{\begin{array}{ll}
L^{2}\left(\Omega, \frac{f(|x|)}{|x|^{N+1}}\right), & N=3,4 ; \\
L^{1}\left(\Omega, \frac{f(|x|)}{|x|^{3} \log (|x|)}\right), & N=2 ;
\end{array} .\right.
$$

For example, one can take $f(r)=\frac{1}{r^{2}}$. Thus $\mathcal{D}_{0}^{2,2}\left(R^{N} \backslash \bar{B}_{1}\right)$ is always a well defined function space.

Remark 3.15. For an annular region $\Omega \subset \mathbb{R}^{4}$, let $g$ be a nonnegative radial function such that $g \in L^{1}(\Omega)$ and $g \notin \mathcal{M} \log L(\Omega)$. Then $g$ is admissible by the above theorem, however $g^{\star}$ is not admissible on $\Omega^{\star}$ by part (ii) of Theorem 1.4. An example of such weight is $g(x)=\frac{1}{\left(|x|^{4}-1\right)\left(\log \frac{16 e}{\left(|x|^{4}-1\right)}\right)^{\frac{3}{2}}}$ on $B(0 ; 2) \backslash B(0 ; 1)$.

Theorem 3.16. Let $\Omega$ be a bounded open set in $\mathbb{R}^{N}$ with $N=1,2$ or 3. Then $g \in L^{1}(\Omega)$ with $g \geq 0$ is admissible.

Proof. For $N=1,2,3, H_{0}^{2}(\Omega)$ is continuously embedded into $L^{\infty}(\Omega)$. Hence, for $g \in$ $L^{1}(\Omega)$,

$$
\int_{\Omega} g u^{2} \mathrm{~d} x \leq\|g\|_{1}\|u\|_{\infty}^{2} \leq C\|g\|_{1} \int_{\Omega}|\Delta u|^{2} \mathrm{~d} x, \forall u \in H_{0}^{2}(\Omega),
$$

where $C$ is the embedding constant. 
Remark 3.17. Let $\Omega$ and $X$ be as in Lemma 3.2 and let

$$
\mathcal{F}_{X}=\overline{\mathcal{C}_{c}^{\infty}(\Omega)} \subset X
$$

Then for $g \in \mathcal{F}_{X}$, the best constant in inequality (1.1) is attained. This will follow as the map $G: \mathcal{D}_{0}^{2,2}(\Omega) \rightarrow \mathbb{R}$ defined by $G(u)=\int_{\Omega} g u^{2} \mathrm{~d} x$ is compact. The compactness of $G$ can be prove using a similar set of arguments as in the proofs of Lemma 15 of [6] and Lemma $5.1[7]$.

\section{Appendix A}

First we see that as a vector space $L^{1, \infty}(\log L)^{2}(\Omega)$ and $\mathcal{M} \log L(\Omega)$ are same.

Proposition A.1. Let $\Omega \subset \mathbb{R}^{N}$ be a bounded open set. Then $L^{1, \infty}(\log L)^{2}(\Omega)=$ $\mathcal{M} \log L(\Omega)$.

Proof. First we show that $\|f\|_{\mathcal{M} \log L} \leq|f|_{(1, \infty, 2)}$. For $f \in \mathcal{M}(\Omega)$ and $t \in(0,|\Omega|)$, we have

$$
\begin{aligned}
t f^{* *}(t) & =\int_{0}^{t} f^{*}(s) \mathrm{d} s=\int_{0}^{t} f^{*}(s) s\left[\log \left(\frac{|\Omega|}{s}\right)\right]^{2} \frac{1}{s\left[\log \left(\frac{|\Omega|}{s}\right)\right]^{2}} \mathrm{~d} s \\
& \leq \sup _{0<s \leq t} f^{*}(s) s\left[\log \left(\frac{|\Omega|}{s}\right)\right]^{2} \int_{0}^{t} \frac{1}{s\left[\log \left(\frac{|\Omega|}{s}\right)\right]^{2}} \mathrm{~d} s \\
& \leq|f|_{(1, \infty, 2)} \frac{1}{\log \left(\frac{|\Omega|}{t}\right)} .
\end{aligned}
$$

This yields $\|f\|_{\mathcal{M} \log L} \leq|f|_{(1, \infty, 2)}$ and hence

$$
L^{1, \infty}(\log L)^{2}(\Omega) \subseteq \mathcal{M} \log L(\Omega) .
$$

If the above inclusion is strict, then $\exists f \in \mathcal{M} \log L(\Omega) \backslash L^{1, \infty}(\log L)^{2}(\Omega)$, i.e.,

$$
\sup _{0<t<|\Omega|} f^{* *}(t) t\left[\log \left(\frac{|\Omega|}{t}\right)\right]<\infty ; \sup _{0<t<|\Omega|} f^{*}(t) t\left[\log \left(\frac{e|\Omega|}{t}\right)\right]^{2}=\infty .
$$

Now consider the function

$$
g(t)=f^{*}(t) t\left[\log \left(\frac{e|\Omega|}{t}\right)\right]^{2}, 0<t<|\Omega| .
$$

Claim: $\lim _{t \rightarrow 0} g(t)=\infty$.

If the claim is not true, then $\exists t_{0}>0$ such that $\sup _{t \geq t_{0}} g(t)=\infty$. Since $t\left[\log \left(\frac{e|\Omega|}{t}\right)\right]^{2}$ is 
bounded, we must have $f^{*}(t)=\infty$ for $t \leq t_{0}$. A contradiction as $f \in \mathcal{M} \log L(\Omega)$ hence claim must be true.

Now by the claim, there exists a decreasing sequence $\left(t_{n}\right)$ in $(0,|\Omega|)$ such that $\left(t_{n}\right)$ converging to 0 and $g(t)>n$, for $t \in\left(0, t_{n}\right)$. Consequently,

$$
t_{n} f^{* *}\left(t_{n}\right)=\int_{0}^{t_{n}} \frac{g(t)}{t\left[\log \left(\frac{e|\Omega|}{t}\right)\right]^{2}} d t \geq n \int_{0}^{t_{n}} \frac{1}{t\left[\log \left(\frac{e|\Omega|}{t}\right)\right]^{2}} d t \geq \frac{n}{\log \left(\frac{e|\Omega|}{t_{n}}\right)} .
$$

Therefore,

$$
\lim _{n \rightarrow \infty} t_{n} f^{* *}\left(t_{n}\right) \log \left(\frac{|\Omega|}{t_{n}}\right) \geq \lim _{n \rightarrow \infty} n \frac{\log \left(\frac{|\Omega|}{t_{n}}\right)}{\log \left(\frac{e|\Omega|}{t_{n}}\right)}=\infty .
$$

A contradiction as $f \in \mathcal{M} \log L(\Omega)$. Hence we must have $L^{1, \infty}(\log L)^{2}(\Omega)=\mathcal{M} \log L(\Omega)$.

Remark A.2. The quasinorm $|f|_{(1, \infty, 2)}$ and the norm $\|f\|_{\mathcal{M} \log L}$ defines the same vector space, however, they are not equivalent. To see this, let $\Omega=B(0 ; R) \subset \mathbb{R}^{N}$ and for each $n \in \mathbb{N}$, consider the function $\left\{f_{n}\right\}$ on $\Omega$ defined as

$$
f_{n}(x)=\frac{1}{|x|^{N}\left[\log \left(\left(\frac{R}{|x|}\right)^{N} e\right)\right]^{n+2}} .
$$

Thus we have $f_{n}^{*}(t)=\frac{\omega_{N}}{t\left[\log \left(\frac{e|\Omega|}{t}\right)\right]^{n+2}}$ and $f_{n}^{* *}(t)=\frac{\omega_{N}}{(n+1) t\left[\log \left(\frac{e|\Omega|}{t}\right)\right]^{n+1}}$. Therefore,

$$
\begin{aligned}
\left|f_{n}\right|_{(1, \infty, 2)} & =\sup _{0<t<|\Omega|} t\left[\log \left(\frac{e|\Omega|}{t}\right)\right]^{2} f_{n}^{*}(t)=\omega_{N} \sup _{0<t<|\Omega|} \frac{1}{\left[\log \left(\frac{e|\Omega|}{t}\right)\right]^{n}} \\
\left\|f_{n}\right\|_{\mathcal{M} \log L} & =\sup _{0<t<|\Omega|} t\left[\log \left(\frac{|\Omega|}{t}\right)\right] f_{n}^{* *}(t) \leq \frac{\omega_{N}}{n+1} \sup _{0<t<|\Omega|} \frac{1}{\left[\log \left(\frac{e|\Omega|}{t}\right)\right]^{n}} .
\end{aligned}
$$

Hence $(n+1)\left\|f_{n}\right\|_{\mathcal{M} \log L} \leq\left|f_{n}\right|_{(1, \infty, 2)}$.

The next proposition provides the equivalence of the quasinorm $|u|_{(\infty, 1,-2)}$ and the norm $\|u\|_{(\infty, 1,-2)}$. We adapt the proof of Theorem 6.4 of [8] to our case.

Proposition A.3. Let $\Omega$ be a bounded subset of $\mathbb{R}^{N}$ and $u: \Omega \rightarrow \mathbb{R}$ be a measurable function. Then there exist a constant $C>0$ such that

$$
\int_{0}^{|\Omega|}\left(\frac{u^{* *}(t)}{\log \left(\frac{e|\Omega|}{t}\right)}\right)^{2} \frac{d t}{t} \leq C \int_{0}^{|\Omega|}\left(\frac{u^{*}(t)}{\log \left(\frac{e|\Omega|}{t}\right)}\right)^{2} \frac{d t}{t}
$$


Proof. Choose $0<\delta<1$ and write $u^{*}(s)=\left[s^{\delta} u^{*}(s)\right]\left[s^{1-\delta}\right] s^{-1}$. Using Holder's inequality we obtain,

$$
\left(\int_{0}^{t} u^{*}(s) d s\right)^{2} \leq C_{1} t^{2-2 \delta}\left(\int_{0}^{t}\left[s^{\delta} u^{*}(s)\right]^{2} \frac{d s}{s}\right) .
$$

Multiplying by $\left(\frac{1}{t^{3}\left(\log \left(\frac{e|\Omega|}{t}\right)\right)^{2}}\right)$ and integrating over $(0,|\Omega|)$ we get

$$
\begin{aligned}
\int_{0}^{|\Omega|}\left(\frac{u^{* *}(t)}{\log \left(\frac{e|\Omega|}{t}\right)}\right)^{2} \frac{d t}{t} & \leq C_{1} \int_{0}^{|\Omega|} \frac{1}{t^{2 \delta}\left(\log \left(\frac{e|\Omega|}{t}\right)\right)^{2}}\left(\int_{0}^{t}\left[s^{\delta} u^{*}(s)\right]^{2} \frac{d s}{s}\right) \frac{d t}{t} \\
& \leq C_{1} \int_{0}^{|\Omega|}\left[s^{\delta} u^{*}(s)\right]^{2}\left(\int_{s}^{|\Omega|} \frac{1}{t^{2 \delta}\left(\log \left(\frac{e|\Omega|}{t}\right)\right)^{2}} \frac{d t}{t}\right) \frac{d s}{s} \\
& \leq C_{1} \int_{0}^{|\Omega|} \frac{\left[s^{\delta} u^{*}(s)\right]^{2}}{s^{\delta}\left(\log \left(\frac{e|\Omega|}{s}\right)\right)^{2}}\left(\int_{s}^{|\Omega|} \frac{d t}{t^{1+\delta}}\right) \frac{d s}{s}
\end{aligned}
$$

The last two inequalities of (A.2) follows from Fubini's theorem and monotonic decreasing property of $\frac{1}{t^{\delta}\left(\log \left(\frac{e|\Omega|}{t}\right)\right)^{2}}$ respectively. Further, we estimate the right hand side of (A.2) as below,

$$
\int_{0}^{|\Omega|} \frac{\left[s^{\delta} u^{*}(s)\right]^{2}}{s^{\delta}\left(\log \left(\frac{e|\Omega|}{s}\right)\right)^{2}}\left(\int_{s}^{|\Omega|} \frac{d t}{t^{1+\delta}}\right) \frac{d s}{s} \leq C_{2} \int_{0}^{|\Omega|} \frac{\left[s^{\delta} u^{*}(s)\right]^{2}}{s^{\delta}\left(\log \left(\frac{e|\Omega|}{s}\right)\right)^{2}}\left(\frac{1}{s^{\delta}}\right) \frac{d s}{s} .
$$

Hence by combining (A.2) and (A.3) we have the following inequality as required

$$
\int_{0}^{|\Omega|}\left(\frac{u^{* *}(t)}{\log \left(\frac{e|\Omega|}{t}\right)}\right)^{2} \frac{d t}{t} \leq C \int_{0}^{|\Omega|}\left(\frac{u^{*}(t)}{\log \left(\frac{e|\Omega|}{t}\right)}\right)^{2} \frac{d t}{t}
$$

\section{References}

[1] D. R. Adams. A sharp inequality of J. Moser for higher order derivatives. Ann. of Math. (2), 128(2):385-398, 1988.

[2] Adimurthi, N. Chaudhuri, and M. Ramaswamy. An improved Hardy-Sobolev inequality and its application. Proc. Amer. Math. Soc., 130(2):489-505 (electronic), 2002.

[3] Adimurthi, M. Grossi, and S. Santra. Optimal Hardy-Rellich inequalities, maximum principle and related eigenvalue problem. J. Funct. Anal., 240(1):36-83, 2006. 
[4] Adimurthi and S. Santra. Generalized Hardy-Rellich inequalities in critical dimension and its applications. Commun. Contemp. Math., 11(3):367-394, 2009.

[5] W. Allegretto. Principal eigenvalues for indefinite-weight elliptic problems in $\mathbf{R}^{n}$. Proc. Amer. Math. Soc., 116(3):701-706, 1992.

[6] T. V. Anoop. A note on generalized Hardy-Sobolev inequalities. Int. J. Anal., 2013:1-9, 2013.

[7] T. V. Anoop, M. Lucia, and M. Ramaswamy. Eigenvalue problems with weights in Lorentz spaces. Calc. Var. Partial Differential Equations, 36(3):355-376, 2009.

[8] C. Bennett and K. Rudnick. On Lorentz-Zygmund spaces. Dissertationes Math. (Rozprawy Mat.), 175:67, 1980.

[9] C. Bennett and R. Sharpley. Interpolation of operators, volume 129 of Pure and Applied Mathematics. Academic Press, Inc., Boston, MA, 1988.

[10] H. Brézis and J. L. Vázquez. Blow-up solutions of some nonlinear elliptic problems. Rev. Mat. Univ. Complut. Madrid, 10(2):443-469, 1997.

[11] H. Brézis and S. Wainger. A note on limiting cases of Sobolev embeddings and convolution inequalities. Comm. Partial Differential Equations, 5(7):773-789, 1980.

[12] A. Cianchi. Second-order derivatives and rearrangements. Duke Math. J., 105(3):355-385, 2000.

[13] A. Cianchi. Symmetrization and second-order Sobolev inequalities. Ann. Mat. Pura Appl. (4), 183(1):45-77, 2004.

[14] A. L. Edelson and A. J. Rumbos. Linear and semilinear eigenvalue problems in $\mathbf{R}^{n}$. Comm. Partial Differential Equations, 18(1-2):215-240, 1993.

[15] D. E. Edmunds and W. D. Evans. Hardy operators, function spaces and embeddings. Springer Monographs in Mathematics. Springer-Verlag, Berlin, 2004.

[16] D. E. Edmunds, R. Kerman, and L. Pick. Optimal Sobolev imbeddings involving rearrangement-invariant quasinorms. J. Funct. Anal., 170(2):307-355, 2000.

[17] D. E. Edmunds and H. Triebel. Sharp Sobolev embeddings and related Hardy inequalities: the critical case. Math. Nachr., 207:79-92, 1999.

[18] S. Filippas and A. Tertikas. Optimizing improved Hardy inequalities. J. Funct. Anal., 192(1):186-233, 2002. 
[19] N. Ghoussoub and A. Moradifam. Bessel pairs and optimal Hardy and Hardy-Rellich inequalities. Math. Ann., 349(1):1-57, 2011.

[20] N. Ghoussoub and A. Moradifam. Functional inequalities: new perspectives and new applications, volume 187 of Mathematical Surveys and Monographs. American Mathematical Society, Providence, RI, 2013.

[21] L. Grafakos. Classical Fourier analysis, volume 249 of Graduate Texts in Mathematics. Springer, New York, second edition, 2008.

[22] K. Hansson. Imbedding theorems of Sobolev type in potential theory. Math. Scand., 45(1):77-102, 1979.

[23] L. Hörmander and J. L. Lions. Sur la complétion par rapport à une intégrale de Dirichlet. Math. Scand., 4:259-270, 1956.

[24] R. A. Hunt. On $L(p, q)$ spaces. Enseignement Math. (2), 12:249-276, 1966.

[25] A. Kufner, L. Maligranda, and L.-E. Persson. The Hardy inequality. Vydavatelský Servis, Plzeň, 2007. About its history and some related results.

[26] G. G. Lorentz. Some new functional spaces. Ann. of Math. (2), 51:37-55, 1950.

[27] A. Manes and A. M. Micheletti. Un'estensione della teoria variazionale classica degli autovalori per operatori ellittici del secondo ordine. Boll. Un. Mat. Ital. (4), 7:285-301, 1973.

[28] M. Milman and E. Pustylnik. On sharp higher order Sobolev embeddings. Commun. Contemp. Math., 6(3):495-511, 2004.

[29] B. Muckenhoupt. Hardy's inequality with weights. Studia Math., 44:31-38, 1972. Collection of articles honoring the completion by Antoni Zygmund of 50 years of scientific activity, I.

[30] R. O'Neil. Convolution operators and $L(p, q)$ spaces. Duke Math. J., 30:129-142, 1963.

[31] F. Rellich. Halbbeschränkte Differentialoperatoren höherer Ordnung. In Proceedings of the International Congress of Mathematicians, 1954, Amsterdam, vol. III, pages 243-250. Erven P. Noordhoff N.V., Groningen; North-Holland Publishing Co., Amsterdam, 1956.

[32] N. M. Stavrakakis and N. Zographopoulos. Global bifurcation results for a semilinear biharmonic equation on all of $\mathbb{R}^{N}$. Z. Anal. Anwendungen, 18(3):753-766, 1999. 
[33] C. Tarsi. Adams' inequality and limiting Sobolev embeddings into Zygmund spaces. Potential Anal., 37(4):353-385, 2012.

[34] A. Tertikas and N. B. Zographopoulos. Best constants in the Hardy-Rellich inequalities and related improvements. Adv. Math., 209(2):407-459, 2007.

[35] N. Visciglia. A note about the generalized Hardy-Sobolev inequality with potential in $L^{p, d}\left(\mathbb{R}^{n}\right)$. Calc. Var. Partial Differential Equations, 24(2):167-184, 2005.

\section{T. V. Anoop}

Department of Mathematics,

Indian Institute of Technology Madras,

Chennai, 600036, India.

Email: anoop@iitm.ac.in

\section{Ujjal Das}

The Institute of Mathematical Sciences, HBNI

Chennai, 600036, India.

Email: ujjaldas@imsc.res.in, ujjal.rupam.das@gmail.com

\section{Abhishek Sarkar}

NTIS, University of West Bohemia

Technická 8, 30614 Plzeň, Czech Republic.

Email: sarkara@ntis.zcu.cz 